\title{
QUALITY ASSESSMENT OF AN EXTENDED INTERFEROMETRIC RADAR DATA PROCESSING APPROACH
}

\author{
W. Tampubolon *, W. Reinhardt \\ AGIS, Institute for Applied Computer Science, Universität der Bundeswehr München, Germany - (winhard.tampubolon, \\ wolfgang.reinhardt)@unibw.de
}

Commission IV, WG IV/3

KEY WORDS: Radar data processing, Interferometry, Phase offset, Quality assessment

\begin{abstract}
:
Radar data acquisition is a reliable technology to provide base data for topographical mapping. Its flexibility and weather independency makes radar data more attractive in comparison with traditional airborne data acquisition. This advantage emplaces radar data acquisition as an alternative method for many applications including Large Scale Topographical Mapping (LSTM). LSTM i.e. larger or equal than 1:10.000 map scale is one of the prominent priority tasks to be finished in an accelerated way especially in third world countries such as Indonesia. The available TerraSAR-X Add on Digital Elevation Model X (TanDEM-X) Intermediate Digital Elevation Model (IDEM) from German Aerospace Center (DLR) as one useful global scientific data set however still complies with High Resolution Terrain Information (HRTI) Level 3 only. The accuracy of the end product of pairwise bi-static TanDEM-X data can be improved by some potential measures such as incorporation of Ground Control Points (GCPs) within the interferometric data processing. It is expected that the corresponding end product can fulfil HRTI Level 4 specification. From this point, we focus on the step of phase difference measurements in radar interferometry to generate elevation model with least square adjustment approach using three main parameters i.e. height reference, absolute phase offset and baseline. Those three parameters are considered to be essential within the Digital Surface Model (DSM) generation process. Therefore it is necessary to find the optimal solution within aforementioned adjustment model. In this paper we use an linearized model, as discussed in section 2.4 , to process the bi-static TanDEM-X datasets and investigate how this improves the accuracy of the generated DSM. As interferometric radar data processing relies on accurate GCP data we use Indonesian Geospatial Reference System (SRGI) for our investigations. Also, we use baseline and phase offset information from TanDEM-X metadata. Subsequently, the DSM generated using Sentinel Application Platform (SNAP) desktop, is the main product used for LSTM. This product has to be assessed using check points derived from conventional airborne data acquisition using RCD-30 metric camera and the accuracy is compared with the accuracy of the IDEM. Summarized, this paper aims on an improvement of the DSM generation by adjusting main parameters through our linearized model.
\end{abstract}

\section{INTRODUCTION}

It is well known, that Satellite-based platforms are very useful for the purpose of geospatial data acquisition. Wide coverage and flexible acquisition modes make satellite-based data very interesting also within topographical base mapping especially for large areas. The role of large scale topographical maps as an essential component of national spatial data infrastructures has been initiated since 2011 by the legislation about Geospatial Information Act in Indonesia. This breakthrough initiated the massive production of large scale topographical maps.

This type of data can support the national development e.g. related to disaster preparedness, detailed spatial planning, etc. However, Large Scale Topographical Mapping (LSTM) is mostly associated with the production of high resolution spatial data which is normally a costly and time consuming process. Currently, in order to provide high resolution 3D geospatial data, large scale topographical mapping uses as data source conventional airborne campaigns.

Since two decades, the acquisition of reliable topographical data also makes use of satellite-based platforms. In specific, Indonesia as one of the archipelagic countries around a disaster prone area requires topographic data and corresponding processing techniques as a framework for supporting disaster preparedness and quick emergency response. Geospatial data are mandatory in this case because they contain fundamental geospatial features especially of the earth surface with proper geometrical accuracies.

During disaster and emergency situations, satellite-based platforms can provide important data and information for decision support systems. As one instance of basic geospatial data, the role of high resolution DSM is essential in order to enable accurate and immediate analysis within quite a number of societal challenges.

In addition, the utilization of geospatial data using topographic maps as a basic reference is mandatory to support regional planning. From this point, the national government of Indonesia encourages rapid mapping activities in a scale of 1:5,000 by local communities. This so called "village mapping" requires high resolution satellite imageries (CSRT) as a primary data source produced within the synchronized national orthorectification program.

To support this program, the presence of both, DSM and GCP, is mandatory as backbone. Therefore, this paper outlines the extended role of GCP as an available nationwide reference data. In other words, we suggest to extend the usage of available GCPs also for DSM generation.

* Corresponding author 


\section{$1.1 \quad$ Large Scale Topographical Mapping}

The recent legislation Act.Nr.4/2011 about Geospatial Information in Indonesia gives exclusive authority to the Geospatial Information Agency of Indonesia (BIG) as the only responsible institution providing official topographic map data of Indonesia. It covers 1.9 million square kilometers land area of Indonesia which is approximately 4 times the land area of Germany.

This governmental act presents an opportunity and a challenge for the geospatial data development especially to support the economic development in Indonesia. In that case, the proper technologies and methodologies have to be integrated to speed up the huge topographic mapping program in various map scales specifically for large scale mapping i.e. equal or larger than $1: 10,000$.

In order to accomplish above mentioned task especially for LSTM, the topographic mapping acceleration program is mandatory. As an example for the 1:5,000 map scale, the number of single map sheets of 2.3 by $2.3 \mathrm{~km}$ size to be produced is 379.014 (Table 1). Giving $10 \%$ priority for cities or built areas, it will end up in 38.000 map sheets. The available capacity allows for an annual production of 100-200 map sheets. It means without any acceleration activities Indonesia will be covered by 1:5,000 topographic maps in more than hundred years.

\begin{tabular}{|r|r|c|r|r|}
\hline & $\begin{array}{c}\text { Map scale } \\
(1: \mathrm{M})\end{array}$ & $\begin{array}{c}\text { Map Coverage } \\
(\text { Length } \times \text { Width }) \\
\text { in Km }\end{array}$ & $\begin{array}{c}\text { Map } \\
\text { Sheets } \\
\text { (Numbers) }\end{array}$ & $\begin{array}{c}\text { Availability } \\
(\%)\end{array}$ \\
\hline 1 & $1,000,000$ & $668 \times 442$ & 37 & 100 \\
2 & 500,000 & $334 \times 221$ & 103 & 100 \\
3 & 250,000 & $167 \times 111$ & 309 & 100 \\
4 & 100,000 & $55.6 \times 55.6$ & 1,245 & 100 \\
5 & 50,000 & $27.8 \times 27.8$ & 3,899 & 100 \\
6 & 25,000 & $13.8 \times 13.8$ & 13,020 & 40 \\
7 & 10,000 & $4.6 \times 4.6$ & 91,547 & 1.5 \\
8 & 5,000 & $2.3 \times 2.3$ & 379,014 & 0.2 \\
9 & 2,500 & $1.15 \times 1.15$ & 880,206 & 0 \\
10 & 1,000 & $0.58 \times 0.58$ & $2,729,319$ & 0 \\
\hline
\end{tabular}

Table 1 Indonesian Topographical Maps Volume (2017)

In this paper, we present an approach of DSM generation for topographical mapping based on IFSAR, which uses GCP data to adjust the main parameters. The role of GCPs is essential in our approach since its applicability and accuracy are mostly the moderate solution for Indonesian region.

IFSAR has the main advantages of all time, all weather operation and cost-effective data acquisition over large areas, especially for those more remote areas. In addition, it can provide topographic information with elevation accuracies comparable to the stereo-photogrammetric approach (Tampubolon, 2015). The sufficient current topographic information as the DSM reference is considerably important for reliable IFSAR DSM generation.

For the purpose of LSTM in Indonesia, it is necessary to provide the Orthorectified Radar Imagery as well as the DSM with sufficient geometric accuracy.

\subsection{Research objectives and motivation}

The main advantage of radar data acquisition is the capability to provide on-demand very high resolution data independent from weather conditions. Frequently, Interferometric Synthetic Aperture Radar (IFSAR) uses active sensors aiming at DSM as well as orthoimage generation.

In general, radar interferometry requires precise/scientific orbital information measured from on-board sensors in order to provide georeferenced data without any field GCP. Unfortunately, due to some errors during data acquisition it is mostly not possible to reach the required accuracy without any field GCPs.

As already presented by Zhou, 2011, the Ice, Cloud and Land Elevation Satellite (ICESat) can be used to calibrate the residuals in IFSAR DSM generation. At this point, we are going further to use more accurate field GCPs as an input to adjust the main parameters in IFSAR DSM generation i.e. height reference, absolute phase offset and baseline value.

In this paper, we aim on extending the method of IFSAR DSM generation to provide a sufficient accuracy for LSTM by only using a minimum amount of GCPs. For this purpose, a linearization of phase offset estimation has been selected as a potential solution to increase the height accuracy.

Our approach needs precise GCP data from GNSS measurements referring to the local height reference system. We also investigate the role of existing phase difference calculations into the height derivation method. Our main goal is to present a more robust approach for the DSM generation based on linear equations for providing above mentioned main parameters.

Our research also focuses on the comparison of different DEM references used for the absolute phase offset estimation. From this, we can select the proper DEM reference, which is adequate enough for our linear model. In order to evaluate the results, it is necessary to compare generated data with various geometric accuracies.

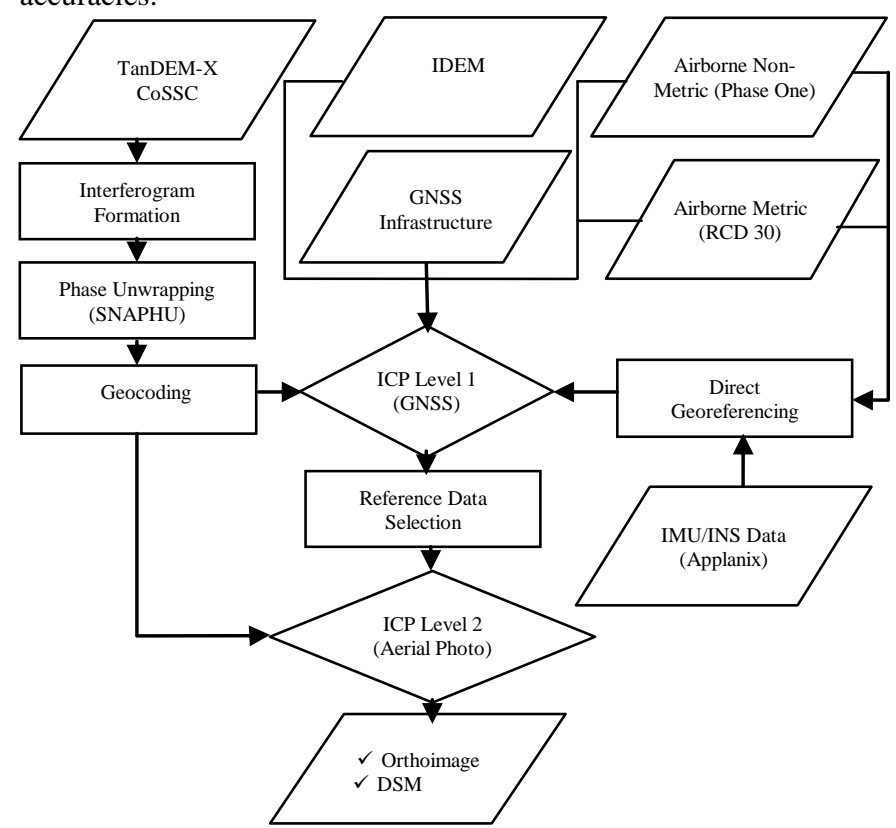

Figure 1. Workflow of our investigations 
We assess our results not only based on the Check Point (CP) data but also based on airborne data equipped with digital medium-format photogrammetric camera as well as a sensor position/orientation measurement unit (Figure 1). The reference data has been selected based on the Independent Check Point (ICP) Level 1 result. The objective of ICP Level 1 is to assess the geometric accuracy for each dataset referring to the GNSS measurements.

Secondly, another objective of our investigations is to define a proper method for the DSM generation from TanDEM-X CoSSC data. A linear approach which allows for a consideration of GCP data has been chosen as an alternative way to derive a DSM with high accuracy. On ICP Level 2, the results from each DSM generation is subsequently validated against reference data acquired from selected reference data either from airborne campaign using Leica RCD30 (metric camera) or Trimble Phase One (P65+). Finally, this research also presents the achievable results for different dataset as described in Table 3.

\subsection{Area of Interest}

We choose our test area around the Geospatial Information Agency of Indonesia (BIG) office in Cibinong because of the availability of reference data, including the geodetic reference network infrastructure with reliable accuracy (see Figure 2).

In general, the test site covers an urban area of approximately $15 \mathrm{~km}^{2}$ which has an approximate elevation of 140 meters above mean sea level (msl). The terrain condition of the study area is classified as medium undulated urban region with a lot of hilly and vegetated areas.

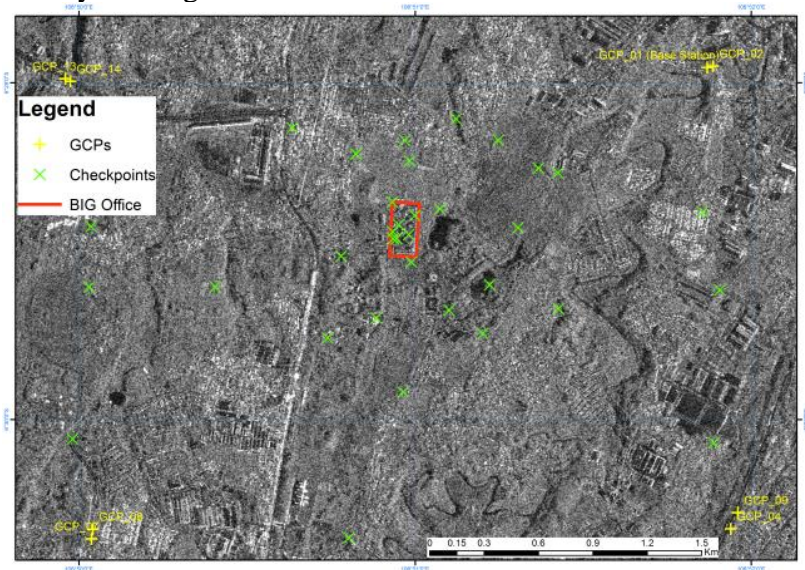

Figure 2. Area of Interest (BIG's office, Indonesia)

Since our algorithm proposes a linear approach to adjust the parameters, we choose only 4 and 8 GCPs configurations around perimeter (Figure 2). In this case, we assume that the GCP distribution will not affect the parameter adjustment results. The most important factor is the GCP's accuracy itself as further explained in 2.3.

\section{CONDUCTED INVESTIGATIONS}

IFSAR DSM generation is a method which provides elevation data from radar datasets only based on phase variation measurements. For the last 10 years, this approach is extensively used not only in the context of aerial acquisition but recently also in conjunction with satellite based acquisition.

\subsection{Data used}

Nowadays, the worldwide user survey among societies has significantly shown that many applications require improved accuracy corresponding to the emerging HRTI standard and hence comparable to the similar DSM generated by airborne Synthetic Aperture Radar (SAR) platform.

After the successful launch of the recent generation of German TerraSAR-X-add-on Digital Elevation Measurement (TanDEM$X)$ satellite in 2010, the ongoing research by using TanDEM-X data was actively initiated by many mapping agencies especially for the DSM generation purpose. This project will hopefully provide the global uniform DSM in a resolution of 10-12 $\mathrm{m}$ in similar way as the SRTM global DEM in 2001.

\begin{tabular}{|l|c|c|c|c|}
\hline Product & \multirow{2}{*}{$\begin{array}{c}\text { Post } \\
\text { spacing } \\
\end{array}$} & \multirow{2}{*}{$\begin{array}{c}\text { Post } \\
\text { spacing } \\
\end{array}$} & $(\mathbf{m})$ & \multicolumn{2}{|c|}{$\begin{array}{c}\text { Accuracies } \\
(\mathbf{9 0 \%} \text { Linear Error) }\end{array}$} \\
\cline { 4 - 5 } & Absolute (m) & Relative (m) \\
\hline DTED Level 0 & 30 & 1,000 & 30 & 20 \\
\hline $\begin{array}{l}\text { DTED Level 1 } \\
\text { and DEM 1 }\end{array}$ & 3 & 100 & 30 & 20 \\
\hline DTED Level 2 & 1 & 30 & 18 & 12 \\
\hline HRTI Level 3 & 0.4 & 12 & 10 & 2 \\
\hline HRTI Level 4 & 0.2 & 6 & 5 & 0.8 \\
\hline HRTI Level 5 & 0.04 & 1 & 2.5 & 0.5 \\
\hline
\end{tabular}

Table 2 Elevation data specification

Referring to the United States Geological Survey (USGS) specification, HRTI stands for High Resolution Terrain Information, while DTED stands for Digital Terrain Elevation Data (Table 2).

In order to get better accuracy, HRTI will be stored as 4-byte (32-bit). However, this will also double the file size compared to using 16-bit data. As an example, HRTI Level 3 requires $2 \mathrm{~m}$ relative vertical accuracy and $10 \mathrm{~m}$ absolute vertical accuracy. Normally, HRTI shall be collected using an airborne IFSAR (Interferometric Synthetic Aperture Radar) platform.

Nevertheless, Intermediate DEM (IDEM) released by TanDEM$\mathrm{X}$ scientific service in 2014 has found its way to comply with the HRTI Level 3 specifications. While using an interferometric approach in the DEM generation, the height calibration still relies on the GCP from ICESat laser altimetry data though.

The IDEM data source will be potentially used for large scale topographical mapping only up to the 1:25.000 map scale. For larger map scales, some necessary improvement shall be included with respect to the available local or ground technical resources and supports.

For our test area, TanDEM-X products i.e. Coregistered Singlelook Slant-range Complex (CoSSC) data have been used as a raw dataset to perform a DSM generation using the interferometric approach (Table 3).

\begin{tabular}{|c|c|c|c|}
\hline Scenes & $\begin{array}{c}\text { HOA*/ } \\
\text { Baseline (m) }\end{array}$ & $\begin{array}{c}\text { Looking } \\
\text { Direction }\end{array}$ & $\begin{array}{c}\text { Acquisition } \\
\text { Date }\end{array}$ \\
\hline S01 & $135.280 / 40.394$ & Descending & $30-01-2012$ \\
\hline S02 & $34.090 / 146.064$ & Descending & $31-10-2012$ \\
\hline S03 & $61.715 / 95.550$ & Ascending & $09-01-2013$ \\
\hline S04 & $120.747 / 52.689$ & Ascending & $11-10-2013$ \\
\hline
\end{tabular}

Table 3. TanDEM-X CoSSC Data (*Height of Ambiguity) 
By using the CoSSC dataset, the interferogram between master and slave channel can be generated directly. However if the coregistration quality is not adequate, we have no chance to modify the transformation parameter of the slave data.

\subsection{Interferometric SAR data processing}

For this paper, we performed the generation of an IFSAR DSM of our test area using TanDEM-X CoSSC data.

For the implementation of our approach, we use the open source Sentinel Application Platform (SNAP) which is the next generation of the Next ESA SAR Toolbox (NEST) focusing on radar interferometry and polarimetry. Fortunately, the TanDEM-X CoSSC (TDM) format can be processed already by the SNAP desktop.

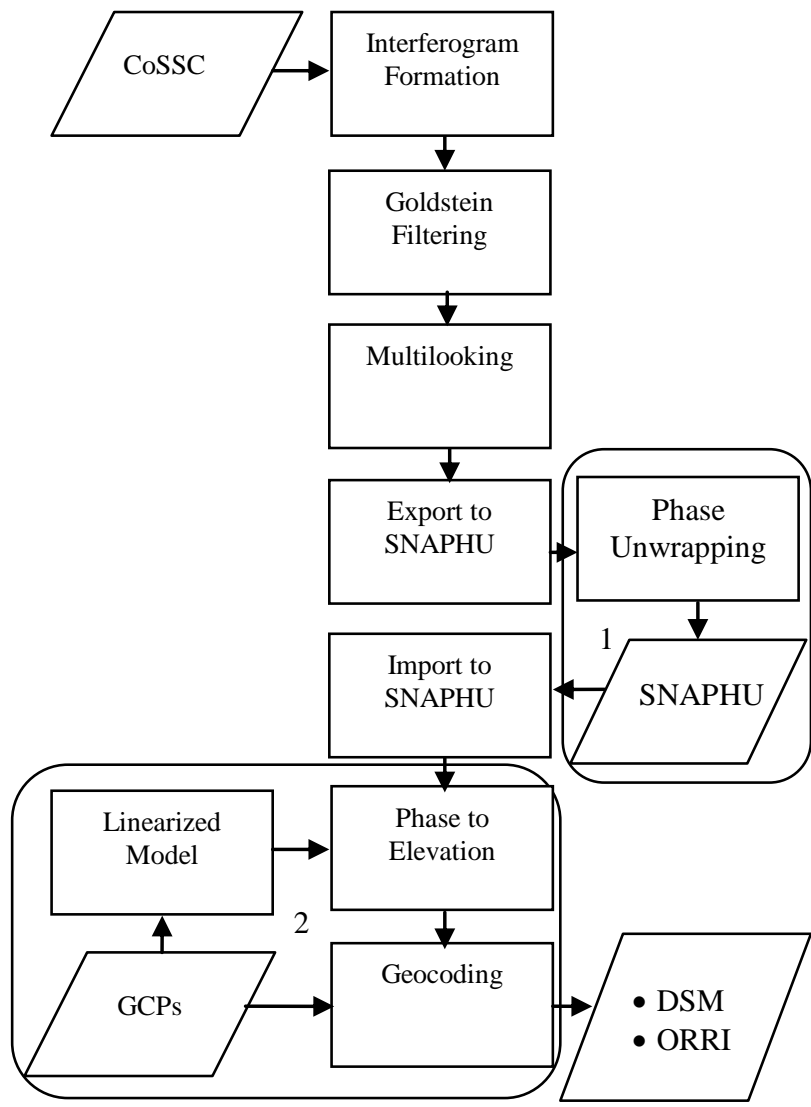

Figure 3. Interferogram formation of CoSSC data

The general steps for IFSAR DSM generation in SNAP desktop are as the following (Veci, 2016):

1. Interferogram formation of the CoSSC data (TDM format) This step provides an interferogram from the pair wise bistatic data acquisition. In order to get only the topographical phase, the flat earth phase must be subtracted.

2. Goldstein filtering

The objective of the Goldstein filtering is to reduce the number of inaccurate fringes from the interferogram.

3. Multilooking

The interferogram multilooking step is necessary to increase the positional accuracy of the intensity and wrapped phase by increasing the number of looking views of the CoSSC data. Normally, 2-5 looking views are the optimal solution to produce effective ground range pixels.

4. Export to SNAPHU (Statistical-Cost Network-Flow Algorithm for Phase Unwrapping)
Currently, the complicated phase unwrapping built-in step is still not provided by the SNAP desktop. Nevertheless, SNAP has an export functionality to hand over the task to the SNAPHU platform.

5. Phase Unwrapping in SNAPHU (Linux-based)

Phase unwrapping using SNAPHU consumes a lot of Random Access Memory (RAM) during processing. Therefore as a rule of thumb, it is necessary to subset the whole area into a size of less than 20 Megabyte of Wrapped Phase Interferograms.

6. Unwrapped Phase to Elevation

The Elevation (height) calculation in SNAP is mainly based on a DEM reference e.g. SRTM 1 Arc Second as an existing topographic phase reference. Hence, the absolute phase offset is determined by the DEM reference accuracy.

7. Geocoding

The geocoding in SNAP considers the terrain correction as well as the GCPs input if available. However, only planimetric $(\mathrm{X}, \mathrm{Y})$ information from the GCPs can be taken into account in the geocoding step.

However, the generated DSM as derived by applying the above mentioned steps is not accurate enough for the LSTM requirements. As a preliminary test in our test area, we get $10.97 \mathrm{~m}$ (95\% height accuracy) of the generated DSM from S01 dataset (Descending-30-01-2012) which is out of the level of the HRTI-3 specification. The main missing part in SNAP desktop is the height calibration based on GCP input. Currently the GCPs are only involved in the geocoding step (Step 7) and not in the absolute phase offset estimation (Step 6).

Therefore, we have developed a height calibration algorithm by taking into account the unwrapped phase as well as the height information from the GCP. As depicted in Figure 3, we focus on 2 components which are SNAPHU Phase Unwrapping (1) and the extension of IFSAR in Phase to Elevation Step (2). There are some pre-condition and consideration for our algorithm:

- The input parameters: Unwrapped Phase, Effective baseline and Initial Phase Offset from TDM metadata.

- The constants : Wavelength $(\lambda)$, Speed of light (C0)

- The phase offset functions : introduced by Mura, 2012

- The GCP data provide initial height reference based on the Indonesian Geospatial Reference System (SRGI)

- Linear adjustment of 3 parameters : Height Reference (href), Absolute Phase Offset $(\Delta \Phi)$, and Baseline $(\Delta \mathrm{BP})$

- Output : Unwrapped Phase, Final Height Reference and adjusted baseline

- Unwrapped Phase to Elevation: Calculated height based on adjusted parameters.

The linear adjustment has been selected because of the flat earth consideration. Finally, we implemented a least square adjustment on our linear height model which will be discussed in section 2.4 .

\subsection{Ground Control Segments}

The Ground control segment is an essential component to achieve the required accuracy. This factor in combination with precise orbital information can provide a robust and sophisticated IFSAR data georeferencing. There are two types of georeferencing applied for our project:

- For georeferencing of all field data, we use the geodetic and geodynamic control of local reference system i.e. Indonesian Geospatial Reference System (SRGI). 
- For on-board georeferencing of the radar data, we use precise/scientific orbital information.

The main reason to use the GNSS monitoring stations (Figure 4) as the positional reference in this project is to ensure the $3 \mathrm{D}$ accuracy. For this purpose, we use Global Navigation Satellite System (GNSS) infrastructure in this case the so called the Continuous Operating Reference System for the global monitoring service, hence the positional accuracy is in the range of millimeter level. Their $2 \mathrm{D}$ positions as well as their height above ellipsoid or mean sea level $(\mathrm{msl})$ using precise geoid model can also be freely accessed online at http://www.srgi.big.go.id.

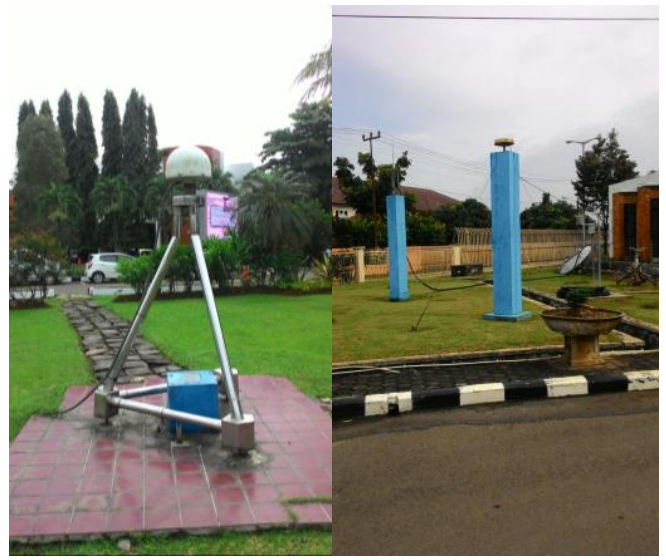

Figure 4. Geodetic Control (CORS) in BIG's office

\subsection{Linearized model in IFSAR DSM generation}

The advent of TanDEM-X with a bi-static interferometry aims on providing HRTI-3 global DEMs which has an accuracy within $10 \mathrm{~m}$ absolute horizontal (circular error) and $10 \mathrm{~m}$ absolute vertical (linear error) at $90 \%$ level of confidence.

TanDEM-X generation implemented data acquisition using bistatic IFSAR approach (DLR, 2012). The basic principle is performing the simultaneous measurement of the same scene and identical doppler spectrum by using 2 sensors, thereby avoiding temporal decorrelation.

In order to produce effective overlap of each doppler spectra, it requires along track baselines less than $2 \mathrm{~km}$ while the across track baselines must be in the range between $300 \mathrm{~m}-1 \mathrm{~km}$. On the other hand, an alternating acquisition mode is also available which allows the simultaneous interferogram generation with single and double baseline of the radar systems (ping pong). The accurate baseline information thereby is really mandatory to produce a high quality DSM.

In addition, errors in the baseline estimation induce a low frequency modulation of the generated DSM, hence affecting the relative and absolute height accuracy. Especially for absolute height accuracy, HRTI Level 4 requirement is stricter and requires an accuracy of $5 \mathrm{~m}$ at a $90 \%$ level of confidence.

The previous baseline phase offset estimation result using phase offset functions (POF) from (Mura, 2012) indicates vertical accuracy in the level of sub meter $(2.768 \mathrm{~m})$ by using OrbiSAR $\mathrm{X}$-Band data. This result motivates us to improve the vertical accuracy in TanDEM-X data by taking into account GCP data in the subsequent linear phase offset estimation using SNAP desktop as was discussed in 2.2.
In our approach, we fixed the height reference as a basis for the absolute height determination within the height calculation. In addition to the flat earth model, the main reason of this selection is that the height reference has its height system locally to the SRGI ground coordinates. This regularity makes an exclusive input to our algorithm with the necessary height calibration at the end.

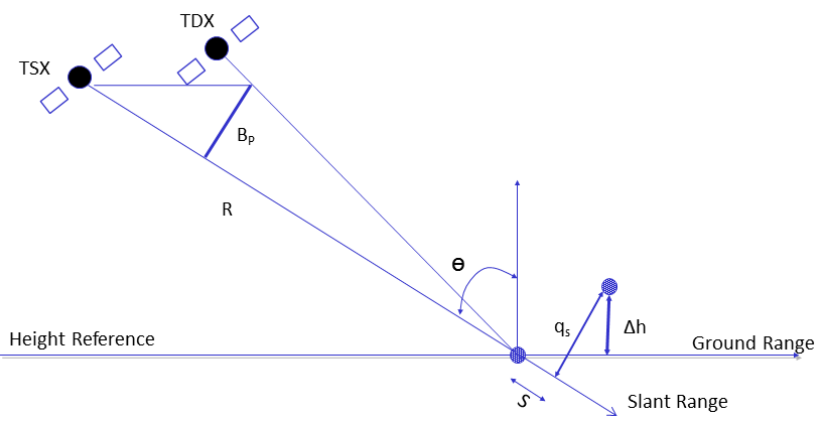

Figure 5. Height calculation consideration

For the DSM generation, the interferometric phase difference is related to the elevation difference in the corresponding measured point on the ground. From the second term in Eq.1, we can get a very important component in the radar interferometry i.e. height ambiguity. As depicted in Figure 5, height ambiguity will determine the relative terrain height $(\Delta \mathrm{h})$ in which it decreases along with increasing perpendicular baseline length $\left(\mathrm{B}_{\mathrm{P}}\right)$.

In order to comply with the HRTI Level 3 standard, it shall be noted that the height ambiguity must be in the order less than 40 $\mathrm{m}$ (Krieger, 2005). In most cases, it is difficult to fulfil this requirement including for our dataset as already explained in 2.1 .

$$
\begin{gathered}
\boldsymbol{h}_{\text {ell }}=\boldsymbol{h}_{\text {ref }}+\frac{\Phi \cdot \lambda_{\cdot} C_{0} \cdot t_{x} \sin \theta}{8 \pi \cdot B_{P}} \\
\boldsymbol{f}(\boldsymbol{t})=\boldsymbol{f}(\boldsymbol{a})+\boldsymbol{f} \cdot(\boldsymbol{t}-\boldsymbol{a})+\frac{\boldsymbol{f}^{n}}{n !}(\boldsymbol{t}-\boldsymbol{a})^{n}
\end{gathered}
$$

Taylor series linearization based on Eq.2 of Eq.1 yields:

$$
\left[\begin{array}{c}
\boldsymbol{h}_{e l l} \\
\vdots \\
\boldsymbol{h}_{\boldsymbol{n}}
\end{array}\right]=\left[\begin{array}{c}
\frac{\Phi \lambda \cdot \mathrm{t} \cdot C_{0} \cdot \sin \theta}{8 \pi \cdot B_{P}} \\
\vdots \\
\frac{\lambda \cdot t_{n} \cdot C_{0} \cdot \sin \theta_{n}}{8 \pi \cdot B_{P}}
\end{array}\right]+\left[\begin{array}{ccc}
\mathbf{1} & \frac{\lambda \cdot C_{0} \cdot t \cdot \sin \theta}{8 \pi \cdot B_{P}} & -\frac{\Phi \cdot \lambda \cdot C_{0} \cdot t \cdot \sin \theta}{8 \pi \cdot B_{P}{ }^{2}} \\
\vdots & \vdots & \vdots \\
\mathbf{1} & \frac{\lambda \cdot C_{0} \cdot \sin \theta_{n}}{8 \pi \cdot B_{P}} & -\frac{\Phi \cdot \lambda \cdot C_{0} \cdot t \cdot \sin \theta_{n}}{8 \pi \cdot B_{P}{ }^{2}}
\end{array}\right]\left[\begin{array}{c}
\boldsymbol{h}_{\boldsymbol{r e f}} \\
\Delta \Phi \\
\Delta \boldsymbol{B}_{P}
\end{array}\right]
$$

From Eq.3, there are three main parameters to be solved in a linear function. With this lightweight linear function, the parameter can be solved and convergents after 4-5 iteration. As an initial input, the effective baseline and phase offset from CoSSC metadata are available. Our linear model is implemented in the Unwrapped Phase to Elevation as already explained in 2.2

\section{RESULTS AND DISCUSSIONS}

In this section, we describe the results of our investigations namely the evaluation of the geometric accuracy and the linear model parameter accuracy.

The selected Ground Sampling Distance (GSD) for orthoimage is $3 \mathrm{~m}$ and $6 \mathrm{~m}$ for the DSM respectively. This resolution allows optimal zooming for manual interpretation and therefore high 
accuracy can be reached for providing 3D reference data in the final evaluation.

A DSM is a representation of the earth surface including manmade and natural structure above ground in three dimensional (3D) coordinates. The derived product which reflects the bare earth is called Digital Terrain Model (DTM). In addition, the Ortho Rectified Imagery (ORI) can be produced by taking into account the DSM (which often is called "True Orthophoto") or the DTM data ("Orthophoto").

With respect to the geometric accuracy, the National Standard for Spatial Data Accuracy (NSSDA) has been selected for geospatial positioning accuracy (FGDC, 1998). The main idea behind this method is the detection of blunders from a given data set and the derivation of a statistical model. In our investigation, we use the Root Mean Square Errors (RMSE) to estimate the accuracy of the different DSMs.

The RMSE can be calculated by the following equation (FGDC, 1998) for each corresponding object in the different datasets i.e. between the IFSAR DSM and the reference data. The calculation focuses on the point features, for the reason of simplicity with high certainty.

$$
\begin{aligned}
& \operatorname{RMSE}_{X}=\sqrt{\frac{\sum\left(X \mathrm{Re}_{i}-\text { XCheck }_{i}\right)^{2}}{n}} \\
& R_{M S E_{Y}}=\sqrt{\frac{\sum\left(Y \mathrm{Re}_{i}-\text { YCheck }_{i}\right)^{2}}{n}} \\
& \operatorname{RMSE}_{r}=\sqrt{\text { RMSE }_{X}^{2}+\text { RMSE }_{Y}^{2}} \\
& \text { Accuracy }_{r}=1.7308 \times R M S E_{r} \\
& \text { RMSE }_{Z}=\sqrt{\frac{\sum\left(Z \mathrm{Re}_{i}-\text { ZCheck }_{i}\right)^{2}}{n}} \\
& \text { Accuracy }_{Z}=1.96 \times \text { RMSE }_{Z}
\end{aligned}
$$

where:

$\mathrm{RMSE}_{\mathrm{x}}=$ Root Mean Square Error in $\mathrm{x}$ axis direction RMSE $_{\mathrm{y}}=$ Root Mean Square Error in y axis direction $\mathrm{RMSE}_{\mathrm{r}}=$ Horizontal (2D) Root Mean Square Error $\mathrm{RMSE}_{\mathrm{Z}}=$ Vertical (3D) Root Mean Square Error

$\left(\mathrm{XRe}_{\mathrm{i}}, \mathrm{YRe}_{\mathrm{i}}, \mathrm{ZRe}_{\mathrm{i}}\right)=$ Coordinates of check-points $\mathrm{i}$ in the reference dataset i.e. Leica RCD30 camera

$\left(\mathrm{XCheck}_{\mathrm{i}}\right.$, YCheck $\left._{\mathrm{i}}, \mathrm{ZCheck}_{\mathrm{i}}\right)=$ Coordinates of check-points $\mathrm{i}$ in the IFSAR DSM (TDX)

$\mathrm{n}=$ number of check-points

The accuracy is given at $95 \%$ confidence level. It means that $95 \%$ of the positions in the dataset will have an error that is equal to or smaller than the reported accuracy value.

ICP Level 1 absolute accuracy assessment for the 2D (planimetric) and 3D (elevation) component has considered 10 checkpoints covering the test area provided from GNSS surveys as included in Table 4. For Direct Georeferencing (DG) from airborne acquisition, we did not use any GCP, while for either Indirect Georeferencing (IG) or combined method we use 3 GNSS monitoring stations and 5 post marking GNSS measurements as 8 GCPs.

Since the DG method is not always free from the systematic errors such as GPS/INS-Camera misalignment, GPS time shift, camera calibration, etc, the combined method using both
GPS/INS data and GCPs is also applied. From ICP Level 1 assessment, we selected DSM from Leica RCD-30 camera with combined georeferencing method as a reference dataset.

\begin{tabular}{|c|c|c|c|c|c|c|}
\hline \multirow{2}{*}{ Camera } & \multicolumn{3}{|c|}{$\begin{array}{c}\text { Planimetric } \\
\text { Accuracy (cm) }\end{array}$} & \multicolumn{3}{c|}{$\begin{array}{c}\text { Elevation } \\
\text { Accuracy (cm) }\end{array}$} \\
\cline { 2 - 7 } & DG & IG & Co & DG & IG & Co \\
\hline $\begin{array}{c}\text { RCD30 } \\
\begin{array}{c}\text { Trimble } \\
\text { Phase } \\
\text { One }\end{array}\end{array}$ & 24.35 & 25.67 & 15.32 & 33.46 & 34.35 & 21.76 \\
\hline
\end{tabular}

Table 4. ICP Level 1 (DG:Direct Georeferencing, IG:Indirect Georeferencing, Co:Combined DG-IG)

As depicted in Figure 6, the profile of building area in BIG office can be better visualized in the generated IFSAR DSM rather than IDEM. However, since the DSM resolution is only 6 $\mathrm{m}$, it is still not sufficient for building extraction purpose.
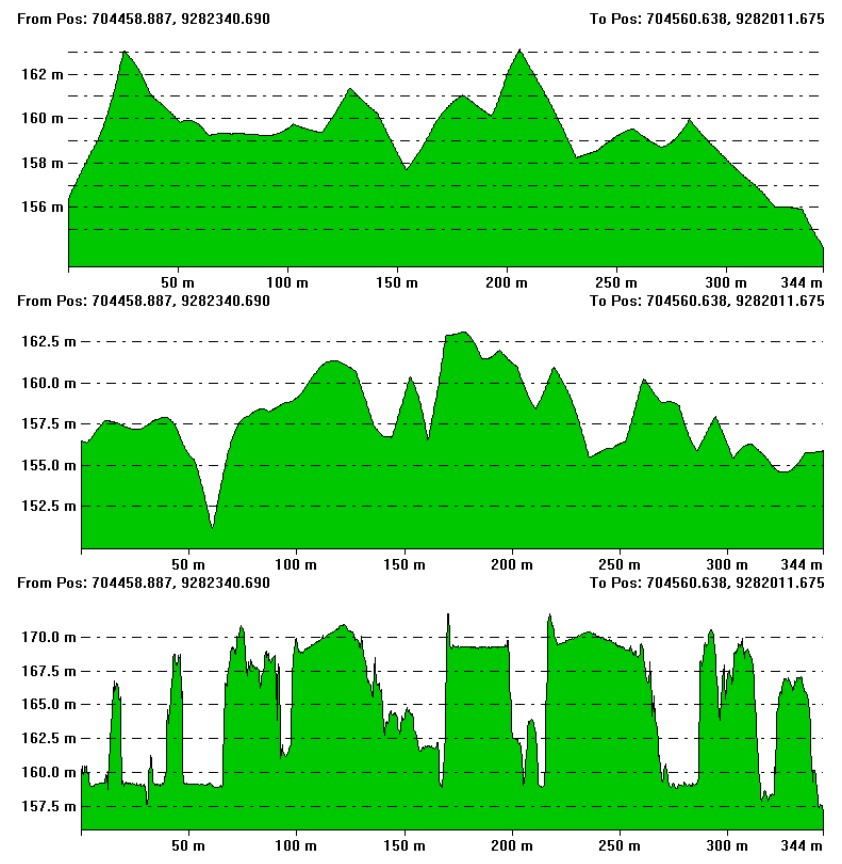

Figure 6. Profile cut in BIG Office: IDEM (upper), Generated DEM (middle) and Leica RCD-30 DSM (lower)

For our appraisal, IFSAR DSM is compared directly with a similar DSM obtained by conventional airborne data acquisition using a Leica RCD30 metric camera (Figure 7). The comparison reveals that our approach can achieve sub meter level accuracy both in planimetric and vertical dimensions.

In addition, ICP Level 2 accuracy assessments have been done by using Leica RCD30 data and 35 Checkpoints as reference data. The generated DSM from IDEM and 4 TDX-CoSSC were validated against Leica RCD30 data in the corresponding resolution. For example to assess the IDEM, we generate the gridded point in $12 \mathrm{~m}$ i.e. 814 points and use ArcGIS tool Extract Values to Points from raster data of Leica RCD30 DSM in $10 \mathrm{~cm}$ resolution. 


\begin{tabular}{|l|c|c|c|c|c|}
\hline & \multirow{2}{*}{$\begin{array}{c}\text { IDEM } \\
(\mathrm{m})\end{array}$} & \multicolumn{4}{|c|}{ TDM IFSAR DSM (m) } \\
\cline { 3 - 6 } & & S01 & S02 & S03 & S04 \\
\hline Without GCP & 6.69 & 10.97 & 12.28 & 17.45 & 23.67 \\
\hline Perimeter GCP (4) & - & 4.38 & 4.67 & 4.75 & 8.49 \\
\hline Perimeter GCP (8) & - & 4.91 & 4.98 & 4.76 & 9.53 \\
\hline
\end{tabular}

Table 5. ICP Level 2 (Final accuracy assessment)

To check the influence of our linear model in our approach, the final assessment was performed. In this case, the results from our approach were directly compared to the conventional generated IFSAR DSM (without GCP). Results from Table 5 also shows that our linear model provides better results on a fewer GCP amount (4). Hence, it confirms the linearity condition of our model perfectly.

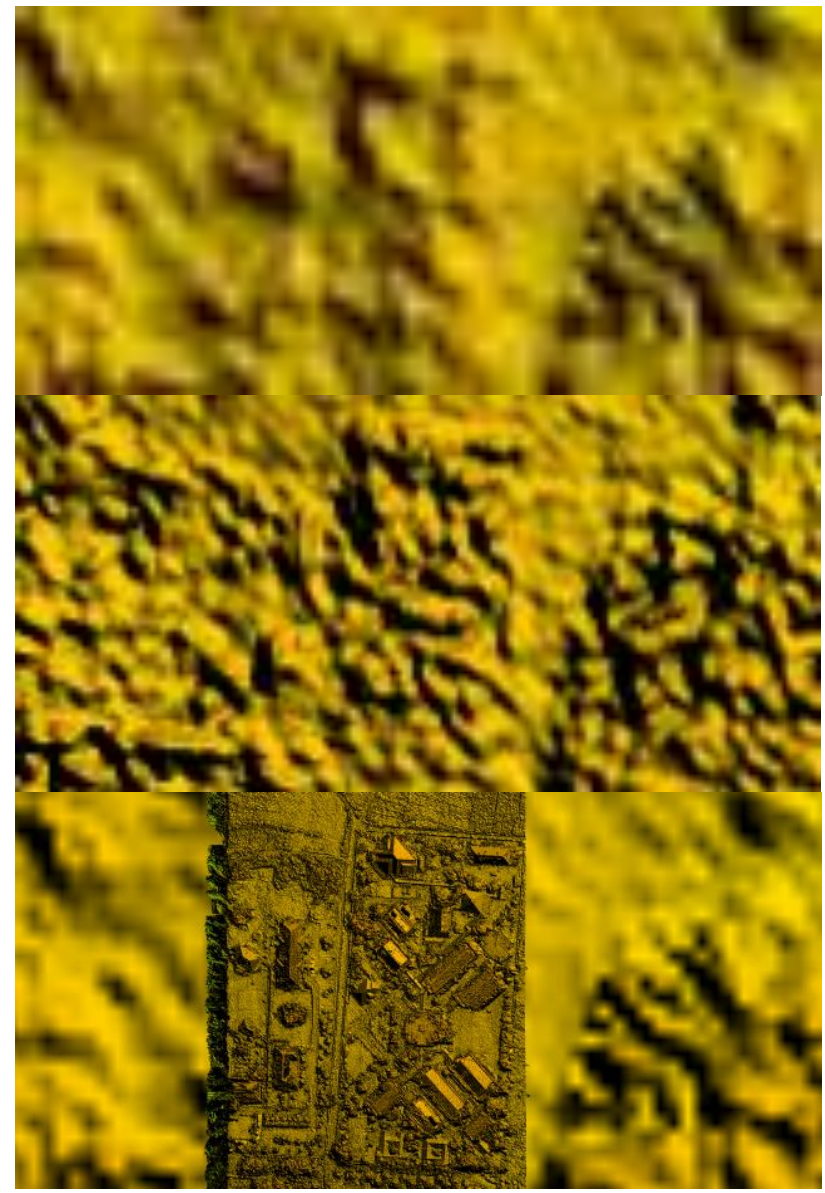

Figure 7 DSM Resolution for the test area: IDEM $12 \mathrm{~m}$ (upper), Generated DEM 6 m (middle) and Leica RCD-30 10 $\mathrm{cm}$ (lower)

However, the unexpected result from the S04 dataset is also occurred. This is mainly coming from the coregistration error in which a lot of incoherent areas are detected in the corresponding Ascending dataset.

\section{CONCLUSIONS}

In this paper, an alternative method to determine the height reference, absolute phase offset and baseline has been presented. The assessment results show that the implementation of our approach for TanDEM-X IFSAR DSM generation can fulfil HRTI Level 4 specification.
This paper also confirmed that IFSAR is a very valuable technique to be utilized in tropical areas though some errors are introduced by layover, foreshortening, shadow, surface decorrelation and the atmospheric signal in the data. GNSS measurement is essential to provide several highly accurate height points for calibrating the residuals in IFSAR DEM. We concluded that the fusion of GCP and IDEM as an intermediate product is necessary to improve the quality of on-going IFSAR DSM generation.

In addition, our approach can generate orthoimage and DSM sufficient enough for 1:10,000 Large Scale Topographical Mapping requirements in Indonesia.

Finally for the future work, since the GCP distribution is not affecting the result, we propose a partial high accuracy DSM from UAV or Aerial Camera as an input for our linear adjustment.

\section{ACKNOWLEDGEMENTS}

We would like to acknowledge German Aerospace Agency (DLR) for providing the scientific TanDEM-X CoSSC, IDEM data and Geospatial Information Agency of Indonesia (BIG) for providing the airborne photogrammetric reference dataset (Leica RCD30, Trimble P65+) as well as the access to the GPS reference station (CORS) during the campaign over the BIG's office in Cibinong, Indonesia.

\section{REFERENCES}

DLR, 2012. TanDEM-X Payload Ground Segment CoSSC Generation and Interferometric Consideration (version 1.0), Oberpfaffenhofen, Germany, TD-PGS-TN-3129, pp. 20-21.

FGDC, 1998. Geospatial Positioning Accuracy Standards Part 3: National Standard for Spatial Data Accuracy (version 2.0), Washington, D.C., FGDC-STD-001-1998: Federal Geographic Data Committee, pp.3-4.

Krieger, G., Fiedler, H., Hajnsek, I., Eineder, M., Werner, M., Moreira, A., 2005, TanDEM-X: Mission Concept and Performance Analysis, Proceedings of IGARSS 2005. Seoul, Korea.

Mura, J.C., Pinheiro, M., Rosa, R., Moreira, J.R., 2012. A Phase-Offset Estimation Method for InSAR DEM Generation Based on Phase-Offset Functions. Remote Sensing Journal, ISSN 2072-4292, pp.745-761.

Tampubolon, W., and Reinhardt, W., 2015. Analysis of Radar and Optical Space Borne Data for Large Scale Topographical Mapping. Int. Arch. Photogramm. Remote Sens. Spatial Inf. Sci., XL-3/W2, pp. 235-242.

Veci, L., 2016. Interferometry Tutorial. Array Systems Computing Inc.,

https://de.scribd.com/document/353837654/S1TBX-StripmapInterferometry-With-Sentinel-1-Tutorial (15 July 2018).

Zhou, Y., Zhou, C., E, D., Wang, Z., Tian, X., 2011. InSAR DEM Reconstruction with ICESAT GLAS Data of the Grove Mountains Area. Fringe 2011 Workshop, Frascati, Italy. 